\title{
Effect of undulator harmonics field on free-electron laser harmonic generation
}

\author{
Qika Jia* \\ National Synchrotron Radiation Laboratory, University of Science and Technology of China, Hefei, Anhui 230029, China
}

(Received 3 November 2010; published 9 June 2011)

\begin{abstract}
The harmonics field effect of a planar undulator on free-electron laser (FEL) harmonic generation has been analyzed. For both the linear case and the nonlinear case, the harmonic fraction of the radiation can be characterized by the coupling coefficients. The modification of the coupling coefficients is given when the third harmonics magnetic field component exists, thus the enhancement of the harmonic radiation can be predicted. The numerical results show that with the third harmonics magnetic field component that has the opposite sign to the fundamental, the intensity of third-harmonic radiation can be increased distinctly for both the small signal gain and the nonlinear harmonic generation. The increase is larger for the smaller undulator deflecting parameter.
\end{abstract}

DOI: 10.1103/PhysRevSTAB.14.060702

PACS numbers: 41.60.Cr, 42.65.Ky, 07.55.Db

\section{INTRODUCTION}

Using the higher harmonic is a way of free-electron laser (FEL) developing towards the shorter wavelength ranges [1-4]. For a planar undulator with an ideal sinusoidal periodic magnetic field, the electrons also radiate at odd harmonics on axis due to their nonuniform axial motion. In actual planar undulators, the magnetic field is nonsinusoidal and with harmonics field components, that has effects on the harmonic radiation. Normally the harmonics fields are very weak. For example, in the hybrid permanent magnet undulator of the Shanghai deep ultraviolet freeelectron laser source [5], the third-harmonic field is less than $1 \%$ of the fundamental field. For a standard Halbachtype pure permanent magnet undulator, which has four magnetic blocks per period, the third-harmonic field may be even weaker. By increasing the harmonic field component aptly, the harmonic radiation can be enhanced [6]. Therefore some methods for this purpose were proposed, such as putting high permeability shims inside the undulator [7], optimizing the magnetic blocks size in a permanent magnet undulator [8]. Ratios of the third harmonics magnetic field to the fundamental magnetic field approaching 30\% have been measured experimentally by Halbach [9]. In this paper, we analyze the effect of undulator harmonics field on FEL harmonic generation, the case of third harmonics magnetic field is considered specially.

\section{ANALYSIS}

In a planar undulator with a sinusoidal periodic magnetic field, the electrons oscillate at odd harmonics

\footnotetext{
*jiaqk@ustc.edu.cn
}

Published by the American Physical Society under the terms of the Creative Commons Attribution 3.0 License. Further distribution of this work must maintain attribution to the author(s) and the published article's title, journal citation, and DOI. frequency in the transverse direction, thus leading to the odd harmonics radiations in the forward direction [10]. For a FEL utilizing such an undulator, the $n$th harmonic optical field equation and the phase equation in one-dimensional mode are [11]

$$
\begin{gathered}
\frac{d}{d z} \tilde{a}_{s n} \simeq \frac{r_{e} n_{e} a_{u}[J, J]_{n} \lambda_{s n}}{\gamma}\left\langle e^{-i n \phi}\right\rangle \\
\frac{d^{2} \phi}{d z^{2}}=-\frac{2 a_{u} k_{u}}{\gamma^{2}} \operatorname{Re} \sum_{n}[J, J]_{n} k_{s n} \tilde{a}_{s n} e^{i n \phi},
\end{gathered}
$$

where $\tilde{a}_{s n}=a_{s n} e^{-i \varphi_{s n}}, a_{s n}=e E_{s n} /\left(m c^{2} k_{s n}\right)$, and $a_{u}=$ $e B_{u} /\left(m c^{2} k_{u}\right)$ are dimensionless vector potential of the rms $n$th harmonic radiation field $E_{s n}$ and undulator field $B_{u}$, respectively; $k_{s n}=2 \pi / \lambda_{s n}, k_{u}=2 \pi / \lambda_{u}$ are the corresponding wave number; $\phi_{s n}$ is the phase of the radiation field; $r_{e}$ is the classical electron radius; $n_{e}$ and $\gamma$ is the density and energy of electrons; $\varphi$ is the ponderomotive phase of electron $\varphi=\left(k_{s}+k_{u}\right) z-\omega_{s} t$. The angular bracket represents the average over the electron's initial phases and initial phase velocities $\varphi_{0}^{\prime} .[J, J]_{n}$ is the coupling coefficient

$$
\begin{aligned}
{[J, J]_{n}=} & (-1)^{(n-1) / 2}\left[J_{(n-1) / 2}\left(\frac{n a_{u}^{2}}{2\left(1+a_{u}^{2}\right)}\right)\right. \\
& \left.-J_{(n+1) / 2}\left(\frac{n a_{u}^{2}}{2\left(1+a_{u}^{2}\right)}\right)\right] .
\end{aligned}
$$

$J$ is integer order Bessel function. From Eqs. (1) and (2) the small signal gain of the $n$th harmonic optical field in the low gain FEL is given by

$$
g_{n}=-n\left(\frac{[J, J]_{n}}{[J, J]_{1}}\right)^{2}(4 \pi N \rho)^{3}\left\langle\frac{\partial}{\partial x} \sin c^{2} \frac{x}{2}\right\rangle_{\phi_{0}{ }^{\prime}},
$$

where $\rho$ is the Pierce parameter, $x=n \varphi_{0}{ }^{\prime} L$, and $L=N \lambda_{u}$ is the length of undulator. In high gain FEL the harmonic is generated nonlinearly. The evolution of the $n$th 
harmonic optical field in the exponential gain region and its saturation power can be given as [11]

$$
\begin{aligned}
\frac{P_{n}}{\rho P_{e}} & \simeq\left(\frac{n^{n-1}[J, J]_{n}}{n ![J, J]_{1}}\right)^{2}\left(\frac{P_{1}}{\rho P_{e}}\right)^{n} \\
& =\left(\frac{n^{n-1}[J, J]_{n}}{n ![J, J]_{1}}\right)^{2}\left(\frac{P_{e f}}{9 \rho P_{e}}\right)^{n} e^{n\left(z / L_{g}\right)} \\
& \frac{P_{n s}}{P_{1 s}} \approx \frac{(n+1)^{n}}{2(n * n !)^{2}}\left(\frac{[J, J]_{n}}{[J, J]_{1}}\right)^{2}
\end{aligned}
$$

where $P_{e}$ is the power of the electron beam; $P_{1}$ and $P_{1 s}$ are the fundamental power and its saturation power, respectively; $P_{e f}$ is the effective start-up short noise power, equal to the fraction of the spontaneous undulator radiation in one power gain length. Thus, the harmonic generation is characterized by the coupling coefficients for both the linear case and the nonlinear case. The coupling coefficients (and consequently the harmonic generation) increase with undulator deflection parameter but the increase becomes very slow after $a_{u}>2$. One can expect an enhancement of the harmonics radiation by adding a harmonic field to the fundamental sinusoidal undulator field.

In actual planar undulators, the magnetic field is nonsinusoidal; when expanded in Fourier series, the field includes odd spatial harmonics due to the symmetry of the magnetic structure. Therefore the magnetic fields and corresponding dimensionless vector potential can be expressed by

$$
\begin{aligned}
B_{u} & =\sum_{m} \sqrt{2} B_{u m} \sin \left(m k_{u} z\right), \\
\tilde{a}_{u} & =\sum_{m} \sqrt{2} a_{u m} \cos \left(m k_{u} z\right),
\end{aligned}
$$

where $m$ is for all or part odd numbers depending on the magnetic structure; $B_{u m}$ and $a_{u m}$ are the rms value of $m$ th harmonics magnetic fields and corresponding dimensionless vector potential, respectively. Generally, all harmonics components are much smaller than the fundamental component:

$$
B_{u m} \ll B_{u 1}, \quad a_{u m}=\frac{B_{u m}}{m B_{u 1}} a_{u 1} \ll a_{u 1} .
$$

Using relation $\beta_{\perp}^{2}=\tilde{a}_{u}^{2} / \gamma^{2}$ and only considering the lower harmonics, the electron longitudinal velocity with the harmonics undulator fields is

$$
\begin{aligned}
\beta_{I I} \approx & 1-\frac{1}{2}\left(\frac{1}{\gamma^{2}}+\beta_{\perp}^{2}\right) \\
= & \bar{\beta}_{\|}-\frac{1}{\gamma^{2}}\left\{\sum_{m} a_{u m}^{2} \cos \left(2 m k_{u} z\right)\right. \\
& \left.+\sum_{m \neq l} a_{u m} a_{u l}\left\{\cos \left[(m+l) k_{u} z\right]+\cos \left[(m-l) k_{u} z\right]\right\}\right\},
\end{aligned}
$$

where $\bar{\beta}_{\|}=1-\frac{1}{2 \gamma^{2}}\left(1+\sum_{m} a_{u m}^{2}\right)$ is the average longitudinal velocity. Accordingly the resonance condition is

$$
\lambda_{s n}=\frac{\lambda_{u}}{2 n \gamma^{2}}\left(1+\sum_{m} a_{u m}^{2}\right)
$$

The longitudinal motion of the electron is

$$
\begin{aligned}
z= & \bar{z}-\left\{\sum_{m} \frac{\xi_{m}}{k_{s}} \sin \left(2 k_{u} \bar{z}\right)+\sum_{m \neq l}\left[\frac{\xi_{m l+}}{k_{s}} \sin \left[(m+l) k_{u} \bar{z}\right]\right.\right. \\
& \left.\left.+\frac{\xi_{m l-}}{k_{s}} \sin \left[(m-l) k_{u} \bar{z}\right]\right]\right\}
\end{aligned}
$$

where $\bar{z}=\bar{\beta}_{\|} c t$,

$$
\begin{aligned}
\xi_{m} & =\frac{a_{u m}^{2}}{2 m\left(1+\sum a_{u i}^{2}\right)}=\frac{r_{m}^{2}}{m} \xi_{1}, \\
\xi_{m l \pm} & =\frac{a_{u m} a_{u l}}{(m \pm l)\left(1+\sum a_{u i}^{2}\right)}=\frac{2 r_{m} r_{l}}{m \pm l} \xi_{1} \\
r_{m} & =\frac{a_{u m}}{a_{u 1}}=\frac{B_{u m}}{m B_{u 1}} \ll 1, \\
\xi_{1} & =\frac{a_{u 1}^{2}}{2\left(1+\sum a_{u i}^{2}\right)}<\frac{a_{u 1}^{2}}{2\left(1+a_{u 1}^{2}\right)}<\frac{1}{2} .
\end{aligned}
$$

For the case that all magnetic harmonic components are much smaller than the fundamental, all the $\xi_{m}, \xi_{m l \pm}$ terms that do not contain the fundamental are much smaller than 1 and can be neglected. Then Eq. (8) becomes

$$
\begin{aligned}
z \simeq \bar{z} & -\left\{\frac{\xi_{1}}{k_{s}} \sin \left(2 k_{u} \bar{z}\right)+\sum_{m \neq 1}\left[\frac{\xi_{m+}}{k_{s}} \sin \left[(m+1) k_{u} \bar{z}\right]\right.\right. \\
& \left.\left.+\frac{\xi_{m-}}{k_{s}} \sin \left[(m-1) k_{u} \bar{z}\right]\right]\right\} .
\end{aligned}
$$

Including the harmonic magnetic fields [Eq. (6)] and with the optical field

$$
\tilde{a}_{s}=\sum_{n} a_{s n} \sin \left[n\left(k_{s} z-\omega_{s} t\right)+\phi_{s n}\right]
$$

the phase equation [Eq. (2)] now is

$$
\begin{aligned}
\phi^{\prime \prime}= & \frac{2 k_{u}}{\gamma^{2}} \sum_{n, l} k_{s n} a_{s n} a_{u l}\left\{\cos \left[\left(n k_{s}+l k_{u}\right) z-n \omega_{s} t+\phi_{n}\right]\right. \\
& \left.+\cos \left[\left(n k_{s}-l k_{u}\right) z-n \omega_{s} t+\phi_{n}\right]\right\} .
\end{aligned}
$$

Substituting Eq. (13) to it, we get 


$$
\phi^{\prime \prime}=\frac{2 k_{u}}{\gamma^{2}} \sum_{n} k_{s n} a_{s n} a_{u 1} f_{n} \operatorname{Re} e^{-i\left(n \phi+\varphi_{s n}\right)},
$$

where

$$
\begin{aligned}
f_{n}= & \operatorname{Re} \sum_{l} \frac{a_{u l}}{a_{u 1}}\left[e^{i(n-l) k_{u} \bar{z}}+e^{i(n+l) k_{u} \bar{z}}\right] e^{i n \xi \sin \left(2 k_{u} \bar{z}\right)} \\
& \times \prod_{m \neq 1} e^{i n\left\{\xi_{m+} \sin \left[(m+1) k_{u} \bar{z}\right]+\xi_{m-} \sin \left[(m-1) k_{u} \bar{z}\right]\right\}} .
\end{aligned}
$$

In obtaining Eq. (17) the condition $k_{s} \gg k_{u}$ is used.

Similarly, when the magnetic harmonic field existed, the $n$th harmonic optical field equation becomes

$$
\frac{d}{d z} \tilde{a}_{s n} \simeq \frac{r_{e} n_{e} a_{u 1} \lambda_{s n}}{\gamma} f_{n}\left\langle e^{-i n \phi}\right\rangle
$$

Comparing Eqs. (16) and (18) with Eqs. (1) and (2), it can be seen that when the magnetic harmonic fields are included the coupling coefficient is modified as

$$
[J, J]_{n} \rightarrow f_{n} .
$$

In the exponential of the modified coupling coefficient [Eq. (17)], many terms are small and oscillate fast; an average over the undulator period will eliminate these small contribution terms.

Among all the harmonics, the third harmonic is the most important one. In the following, we consider the case that only the third-harmonic field exists, and all other harmonics are neglected. Then Eq. (13) can be written as

$$
z=\bar{z}-\frac{\zeta_{1}}{k_{s 1}} \sin \left(2 k_{u} \bar{z}\right)-\frac{\zeta_{2}}{k_{s 1}} \sin \left(4 k_{u} \bar{z}\right)
$$

where

$$
\begin{aligned}
& \xi_{1}=\frac{k_{s 1} a_{u 1}^{2}}{4 k_{u} \gamma^{2}}\left(1+\frac{a_{u 3}}{a_{u 1}}\right)=\frac{a_{u 1}\left(a_{u 1}+a_{u 3}\right)}{2\left(1+a_{u 1}^{2}+a_{u 3}^{2}\right)}, \\
& \xi_{2}=\frac{k_{s 1} a_{u 3} a_{u 1}}{8 k_{u} \gamma^{2}}=\frac{a_{u 1} a_{u 3}}{4\left(1+a_{u 1}^{2}+a_{u 3}^{2}\right)},
\end{aligned}
$$

and it has

$$
\frac{\zeta_{2}}{\zeta_{1}}=\frac{a_{u 3} / a_{u 1}}{2\left(1+a_{u 3} / a_{u 1}\right)}=\frac{B_{u 3} / B_{u 1}}{6+2 B_{u 3} / B_{u 1}} \ll 1 .
$$

Then the modified coupling coefficient [Eq. (17)] is

$$
\begin{aligned}
f_{n}= & \sum_{l=1,3} \frac{a_{u l}}{a_{u 1}}\left[e^{i(n-l) k_{u} \bar{z}}+e^{i(n+l) k_{u} \bar{z}}\right] \\
& \times \sum_{h_{1}} \sum_{h_{2}} J_{h_{1}}\left(n \zeta_{1}\right) J_{h_{2}}\left(n \zeta_{2}\right) e^{i\left(h_{1}+2 h_{2}\right) 2 k_{u} \bar{z}} .
\end{aligned}
$$

Here $n=1,3$. After an average over the undulator period, the dominant product terms in the sum of Eq. (21) are those with $h_{1}+2 h_{2}=-(n \pm l) / 2$ :

$$
\begin{aligned}
f_{n}= & \sum_{l} \frac{a_{u l}}{a_{u 1}}\left\{\sum_{\substack{h_{1}, h_{2}, h_{1}+2 h_{2}=-(n+1) / 2}} J_{h_{1}}\left(n \zeta_{1}\right) J_{h_{2}}\left(n \zeta_{2}\right)\right. \\
& \left.+\sum_{\substack{h_{1}, h_{2} 2 \\
h_{1}+2 h_{2}=-(n-l) / 2}} J_{h_{1}}\left(n \zeta_{1}\right) J_{h_{2}}\left(n \zeta_{2}\right)\right\} \quad n, l=1,3 .
\end{aligned}
$$

For small arguments, only Bessel functions of zero order will contribute. Because $\zeta_{2} \ll \zeta_{1}<1 / 2$, the above equation can be further simplified by taking $h_{2}=0$. Then, at last, we give the modified coupling coefficient as

$$
f_{1}=J_{0}\left(\zeta_{2}\right)\left\{\left[J_{0}\left(\zeta_{1}\right)-J_{1}\left(\zeta_{1}\right)\right]+\frac{a_{u 3}}{a_{u 1}}\left[J_{2}\left(\zeta_{1}\right)+J_{1}\left(\zeta_{1}\right)\right]\right\}
$$

$$
\begin{aligned}
f_{3}= & J_{0}\left(3 \zeta_{2}\right)\left\{\left[J_{2}\left(3 \zeta_{1}\right)-J_{1}\left(3 \zeta_{1}\right)\right]\right. \\
& \left.+\frac{a_{u 3}}{a_{u 1}}\left[J_{0}\left(3 \zeta_{1}\right)-J_{3}\left(3 \zeta_{1}\right)\right]\right\} .
\end{aligned}
$$

According to the above formulas, the modified harmonic coupling coefficients as a function of undulator parameter are numerically calculated for different harmonic magnetic field fraction. The results reveal that the harmonic coupling coefficients and, consequently, the harmonic emission are enhanced when $B_{u 3}$ has an opposite sign to $B_{u 1}$, and are suppressed when the magnetic fields have the same sign (Fig. 1). The results also show that the fundamental coupling coefficient has been less affected by harmonic magnetic field (Fig. 2).

For both the small signal gain and the nonlinear harmonic generation in high gain, the harmonic FEL radiation is proportional to the square of the coupling coefficient [Eqs. (4)-(6)]. Therefore comparing with the case without the harmonic magnetic field present, the enhancement of the 3rd harmonic radiation can be given as

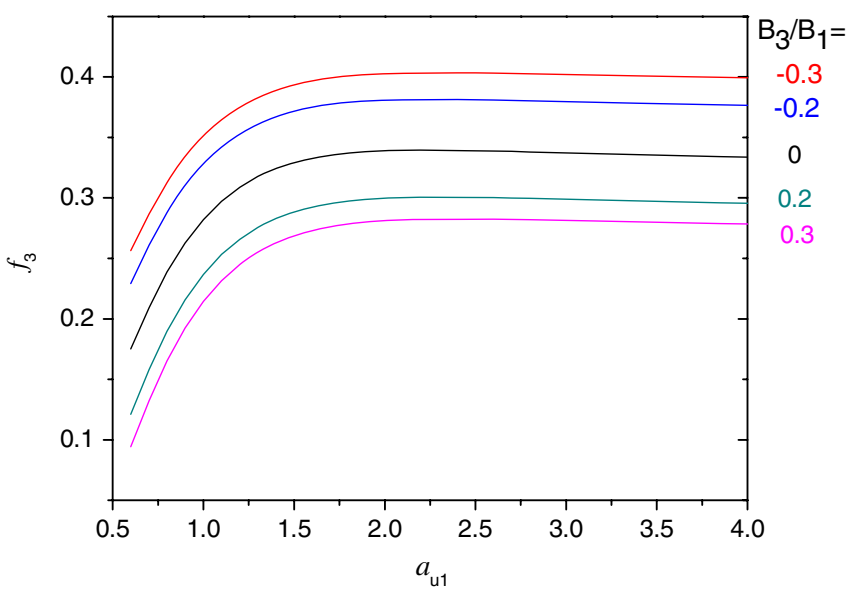

FIG. 1. Modified third-harmonic coupling coefficient due to the harmonic magnetic field. 


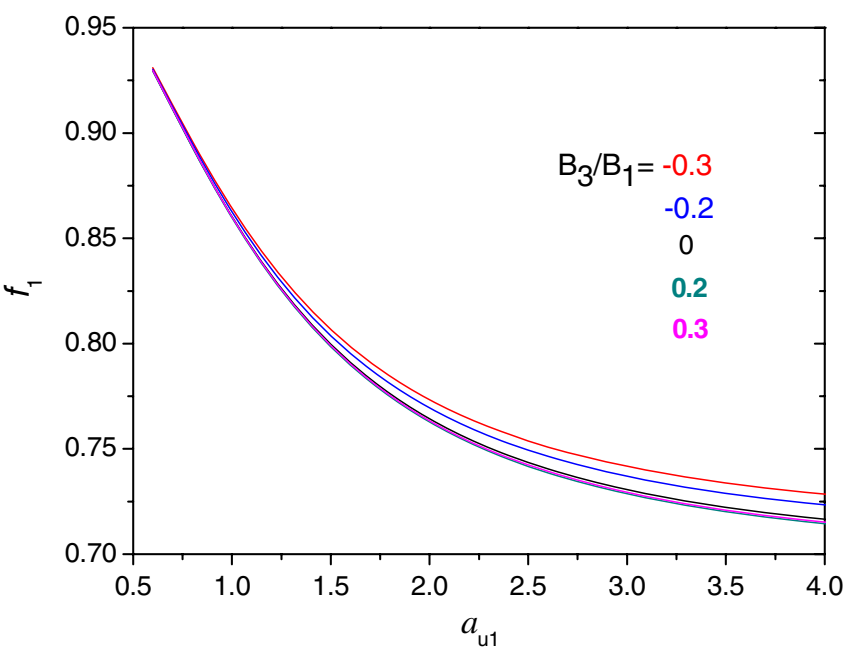

FIG. 2. Modified fundamental coupling coefficient due to the harmonic magnetic field.

$$
R_{3}=\left(\frac{f_{3} / f_{1}}{[J, J]_{3} /[J, J]_{1}}\right)^{2} .
$$

The dependence of the third-harmonic radiation on the ratio of $B_{u 3}$ to $B_{u 1}$ is shown in Fig. 3. The enhancement of the third-harmonic radiation is shown in Fig. 4. While in the calculation of Eq. (25), the arguments of the coupling coefficients and of the modified coupling coefficients are taken with a little difference to keep the same resonant wavelengths, it is $a_{u}$ in the former, and $a_{u 1}$ in the latter:

$$
a_{u}^{2}=a_{u 1}^{2}\left[1+\left(\frac{a_{u 3}}{a_{u 1}}\right)^{2}\right] .
$$

We can see that the harmonics radiation enhancement increases with the magnetic field ratio of the harmonics to the fundamental, and for a given harmonic magnetic field fraction, the enhancement is larger when the magnetic field is weaker. With the third harmonics magnetic field $30 \%$ of the fundamental, this field ratio translates into a vector

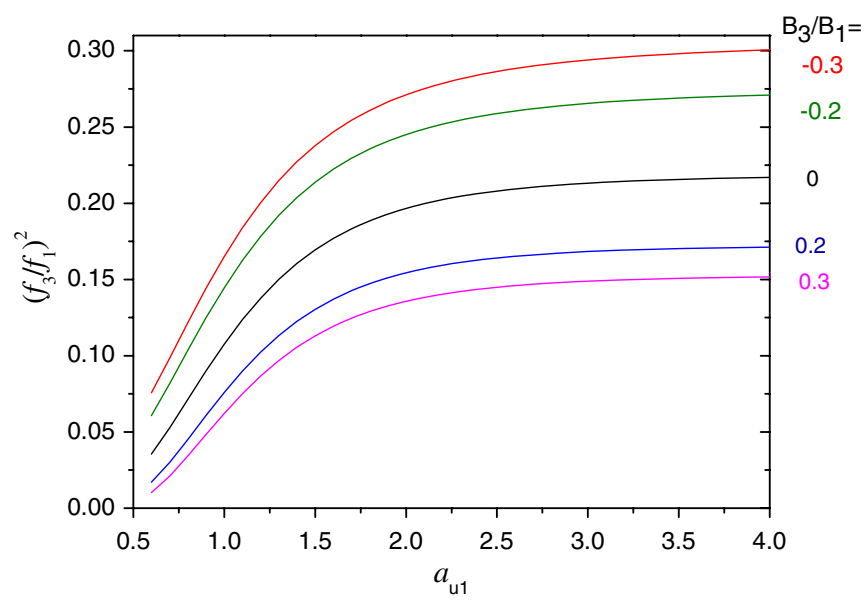

FIG. 3. The effect of $B_{3}$ on FEL harmonic generation.

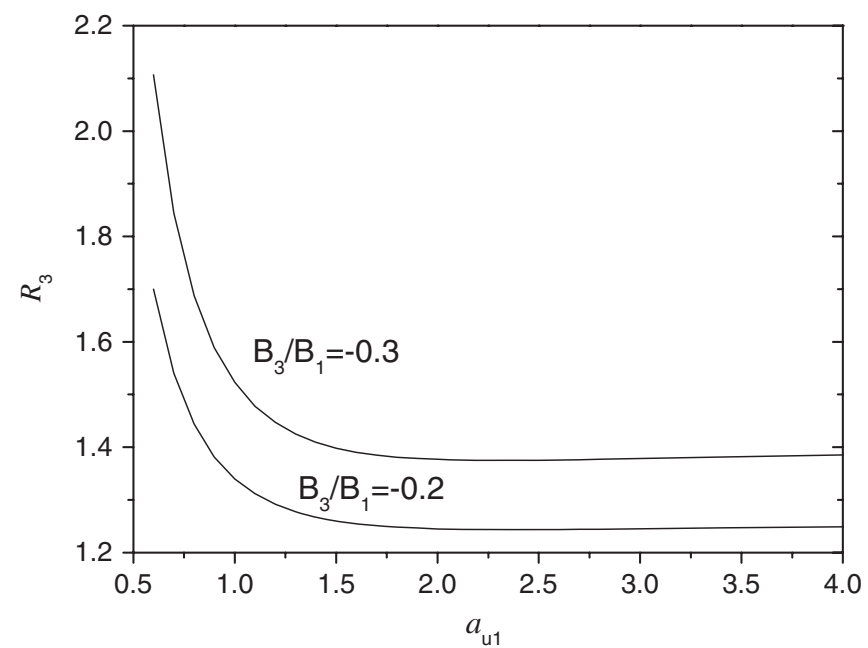

FIG. 4. The enhancement of the FEL harmonic radiation with $B_{3}$.

potential ratio of $10 \%$, the intensity increase of the thirdharmonic radiation is about $40 \%$, and becomes larger when the undulator deflecting parameter is small; for example, it is doubled for undulator deflecting parameter $K\left(=\sqrt{2} a_{u}\right)=0.9$. For $75 \%$ enhancement of the thirdharmonic power, the corresponding third-harmonic coupling coefficient is increased one-third, which agrees with the result of Ref. [6].

Replacing the coupling coefficients with the modified ones, Eqs. (1) and (2) were numerically solved. The parameters we used were based on that of the Hefei soft x-ray FEL proposal [12]. The electron beam parameters were energy of $800 \mathrm{MeV}$, initial energy spread of $0.01 \%$, emittance of $2.1 \mathrm{~nm}-\mathrm{rad}$, and current of $600 \mathrm{~A}$. The undulator parameter $K$ is 1.2 with the period of $2.5 \mathrm{~cm}$. Figure 5

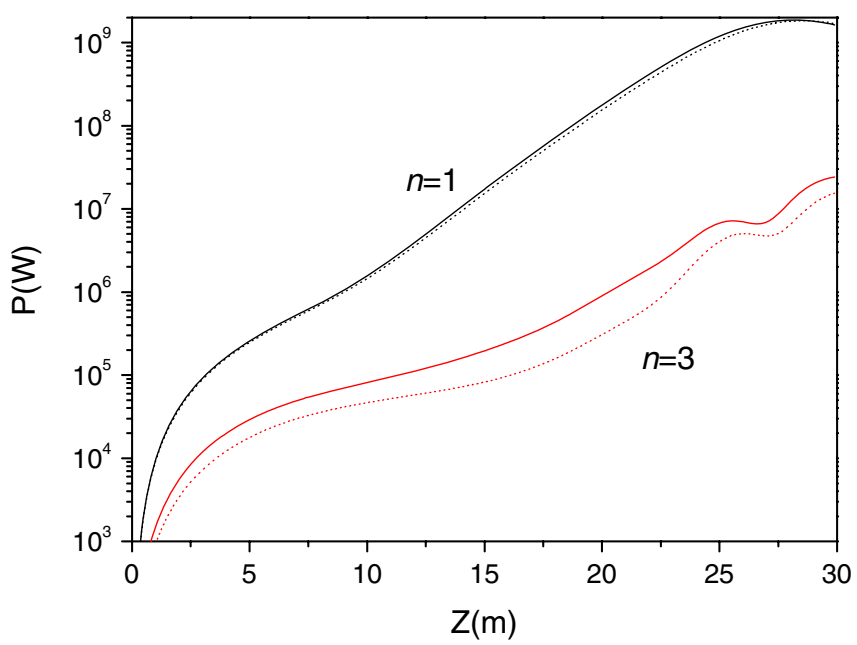

FIG. 5. The nonlinear harmonic generation in SASE FEL with (solid line) and without (dashed line) third harmonics magnetic field. The third harmonics magnetic field $30 \%$ of the fundamental and with an opposite phase to the fundamental is considered. 


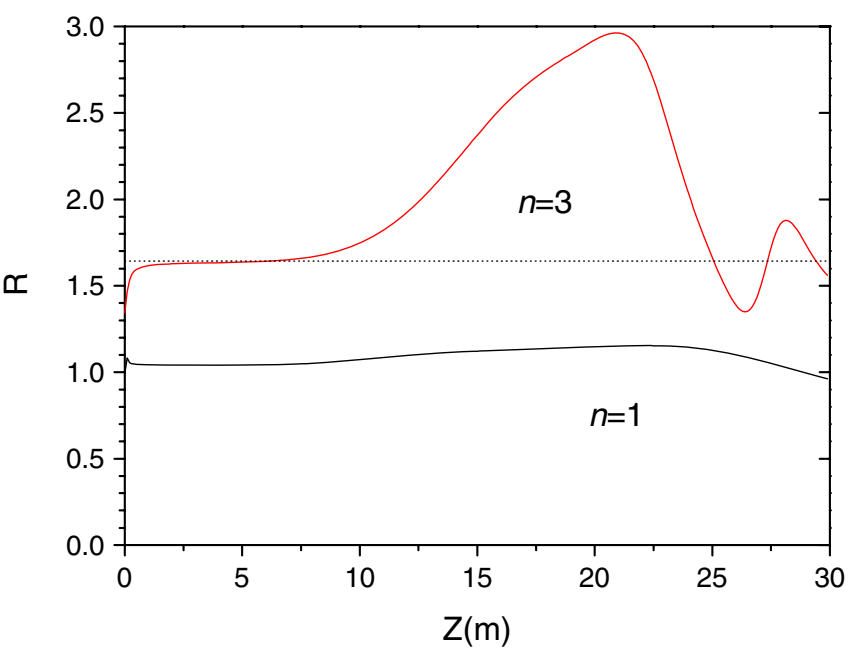

FIG. 6. The enhancement of the harmonic radiation [dashed line: result of Eq. (25)].

shows the effect of the third-harmonic magnetic field on evolution of the fundamental and the third-harmonic radiations. The case of the third-harmonic magnetic field being $30 \%$ of the fundamental is considered. It can be seen that comparing with the case without third-harmonic magnetic field present, the third-harmonic radiation was increased distinctly. The enhancement of the third harmonic radiation agrees well with our previous analytic analysis in the low gain region, is large than the analytic analysis in the exponential gain region, and waves around the analytic value in the saturation region (Fig. 6). Similar results can be obtained with other sets of parameters. The analytic results were given by Eq. (25), in the small signal region it is derived from Eq. (4), a strict formula; in the saturation region it is derived from Eq. (6), an approximate formula; in the exponential gain region it is derived from Eq. (5) but neglected that the gain length $L_{g}$ also were changed with the coupling coefficient.

\section{SUMMARY}

In summary, we have analyzed the effects of the undulator harmonics field on the coupling coefficients and FEL harmonic generation. For the case where the thirdharmonic field is present, analytical expressions of the modified coupling coefficients are given; they can be easily calculated to predict the effects of the undulator harmonics field on both the small signal gain in low gain FEL and the nonlinear harmonic generation in high gain FEL. The numerical results demonstrate that the third-harmonic emission can be distinctly enhanced by the undulator third-harmonic field that has an opposite sign to the fundamental, while the fundamental emission has been less affected. With a third-harmonic field $30 \%$ of the fundamental field, the third-harmonic emission can be enhanced about $40 \%$, and even be doubled for the smaller value of the undulator deflecting parameter.

In addition, since the spontaneous radiation, i.e., the undulator radiation in synchrotron radiation light source, also relates to the coupling coefficients, therefore the similar analysis may be adopted and will be helpful for using the harmonic magnetic fields to enhance or suppress the harmonic undulator radiation.

\section{ACKNOWLEDGMENTS}

This work was supported by the National Nature Science Foundation of China under Grant No. 10975137.

[1] W. B. Colson, IEEE J. Quantum Electron. 17, 1417 (1981).

[2] R. Bonifacio, L. De Salvo, and P. Pierini, Nucl. Instrum. Methods Phys. Res., Sect. A 293, 627 (1990).

[3] H. P. Freund, S. G. Biedron, and S. V. Milton, Nucl. Instrum. Methods Phys. Res., Sect. A 445, 53 (2000).

[4] Z. Huang and K.-J. Kim, Phys. Rev. E 62, 7295 (2000); Nucl. Instrum. Methods Phys. Res., Sect. A 475, 112 (2001).

[5] Jia Qika et al., Proceedings of the FEL 2004 Conference (Comitato Conferenze Elettra, Trieste, Italy, 2004), pp. 494-497.

[6] M. J. Schmitt and C. J. Elliott, IEEE J. Quantum Electron. 23, 1552 (1987).

[7] M. Asakaw et al., Nucl. Instrum. Methods Phys. Res., Sect. A 358, 399 (1995); 375, 416 (1996).

[8] Qi-ka Jia, Proceedings of IPAC10, WEPD033/3165-3167.

[9] K. Halbach, J. Phys. (Paris) 44, C1-211 (1983).

[10] Qi-ka Jia, in Proceedings of the 23rd Particle Accelerator Conference, Vancouver, Canada, 2009 (IEEE, Piscataway, NJ, 2009), WE5RFP088.

[11] Qi-ka Jia, IEEE J. Quantum Electron. 43, 833 (2007).

[12] Concept Design Report of Hefei Soft X-ray FEL Facility, NSRL (2006) (in Chinese). 